\title{
Diffuse lipoatrophic panniculitis: A disfiguring disease
}

\author{
FZ Elfatoiki ${ }^{1}$, MD Vignon Pennamen ${ }^{2}$, V Saada ${ }^{1}$, A Petit ${ }^{1}$, M Bagot ${ }^{1}$, F Cordoliani $^{1}$ \\ 1. Department of Dermatology, University of Paris Diderot, Sorbonne Paris Cité, AP-HP, Saint Louis Hospital, Paris, France. \\ 2. AP-HP, Saint-Louis hospital, Pathology Laboratory, Paris, France.
}

Correspondence: Fatima-zahra Elfatoiki. Address: Department of Dermatologie, University of Paris Diderot, Sorbonne Paris Cité, AP-HP, Saint Louis Hospital, Paris, France. Email: Fatiza59@hotmail.fr

Received: March 4, 2014

DOI : $10.5430 /$ crim.v1n2p191

Online Published: J une 25, 2014

Accepted: May 26, 2014

im.v1n2p191

\section{Abstract}

Lipoatrophic panniculitis is a rare disease in which atrophy of the subcutaneous fat is the main residual lesion. We report the case of a 49-year-old woman without evidence of any associated connective tissue diseases, with a four months history of repeated painful and erythematous nodules and plaques of lower limbs that resolved leaving depressed areas of the skin. Cutaneous biopsy showed lymphohistiocytic lobular panniculitis. Our patient responded well to a combination of prednisone and hydroxychloroquine.

\section{Keywords}

Panniculitis, Lipoatrophy, Lipophagic granulomatous

\section{Introduction}

Lipoatrophic panniculitis is a rare disease that usually affects children and characterized by eruption of tender erythematous nodules and plaques principally of the arms and legs that leave residual circumferential bands of lipoatrophy. The histological feature includes lipophagic panniculitis with absence of vasculitis. This disease may resemble many conditions including connective tissue panniculitis with which it has a nosological relationship. We report a case of diffuse and symmetrical lipoatrophic panniculitis of the lower limbs in 49-year-old woman .

\section{Case report}

A 49-year-old woman presented with a four-month history of repeated painful and erythematous nodules of lower limbs centifugally expanding, leaving central depressed areas. This was preceded by an unexplained oedema of the legs. She had no fever, no systemic symptoms and no familiar history of lypoatrophic panniculitis.

Cutaneous examination showed symmetrical inflammatory plaques of the legs and lower part of the thighs with an erythematous indurated border and central lipoatrophy (see Figure 1). A non-pitting edema of the legs was present. General examination was normal.

A first biopsy specimen from the inflammatory border showed a non specific lobular panniculitis without vasculitis. The infiltrate was mainly composed of lymphocytes and lipophagic histiocytes (see Figure 2). Two biopsies obtained three 
weeks further yielded the same results. Laboratory results showed elevated erythrocyte sedimentation rate $(101 \mathrm{~mm} / \mathrm{h})$ and C-reactive protein level $(44 \mathrm{mg} / \mathrm{l})$, as well as lymphopenia $\left(7 \times 10^{9} / \mathrm{l}\right)$. Serum muscle enzymes (CPK, aldolase), liver enzymes (AST, ALT), LDH, alkaline phosphatase, amylase, lipase and $\alpha 1$-antitrypsin were in a normal range. Immunologic tests including immunoelectrophoresis, antinuclear antibodies (ANA), anti-ds-DNA, rheumatoid factor, lupus anticoagulant, anticardiolipin antibodies were negative. C4 and C3 level were normal. Serologic test for HIV, HBV, $\mathrm{HCV}$ and Lyme disease were negative.

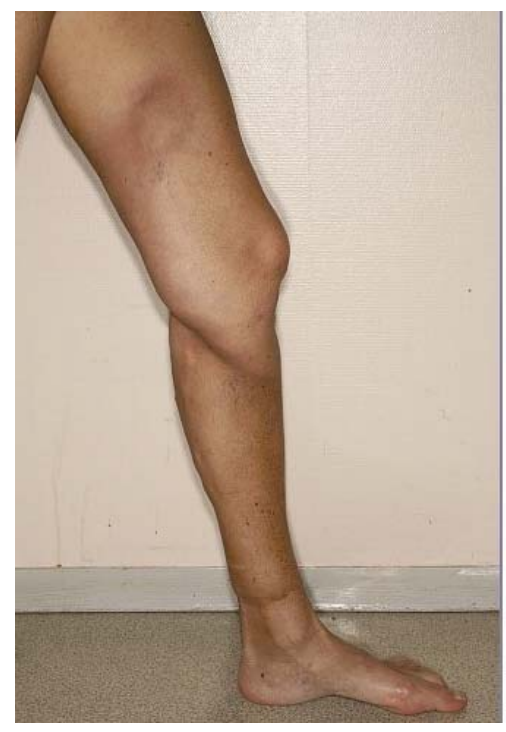

Figure 1. Centrifugally enlarging inflammatory plaque of the left thigh
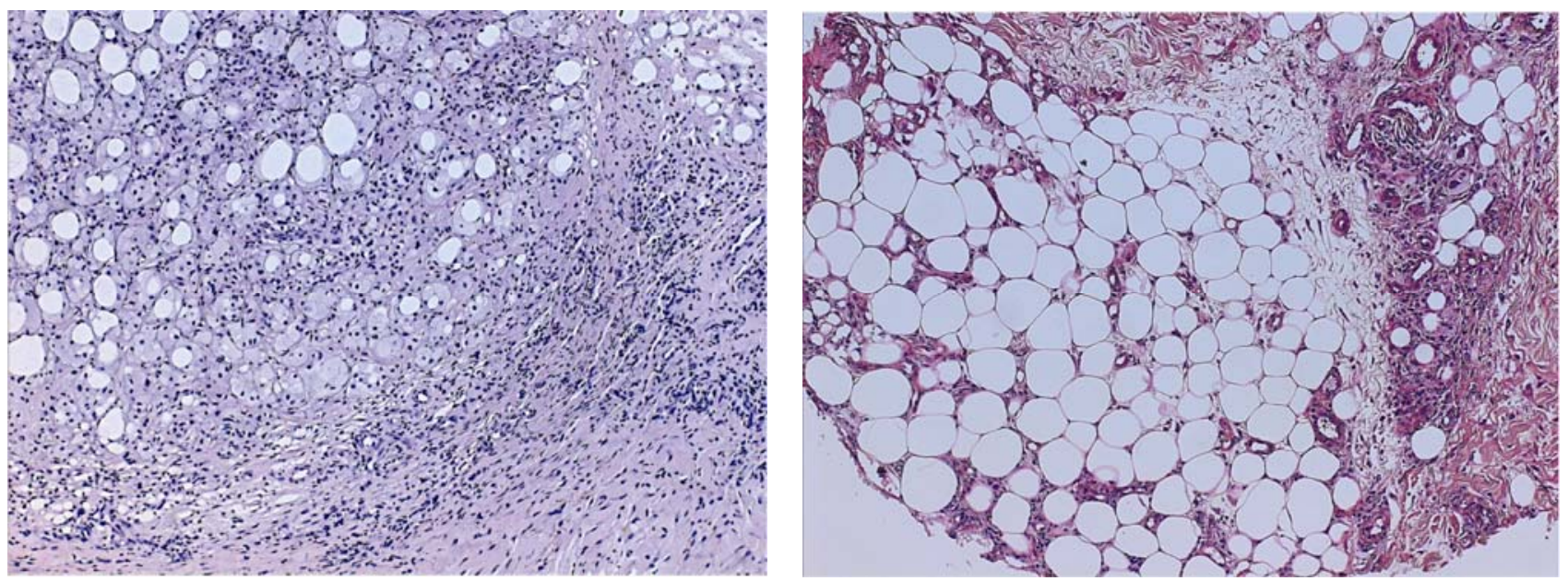

Figure 2. Histopathological features

The patient began treatment with hydroxychloroquine $(200 \mathrm{mg} / \mathrm{d})$, colchicine $1 \mathrm{mg} / \mathrm{d}$ and clobetasol dipropionate cream with moderate improvement, then prednisone $(1 \mathrm{mg} / \mathrm{kg} / \mathrm{d})$ was added with disappearance of inflammation after 15 days, leaving circumferential lipoatrophy of the legs and lower part of the thighs and localized patches of lipoatrophy of the upper part of the thighs. When prednisone was tapered to $10 \mathrm{mg} / \mathrm{d}$, an inflammatory nodule appeared on the spared part of the ventral aspect of the thighs followed very rapidly by others, the patient had unfortunately stopped the treatment without medical advice. Predisone was reintroduced with a very rapid disappearance of inflammation leaving new lipoatrophic areas of the thighs (see Figure 3) and methotrexate was further added as a steroid-sparing agent. 


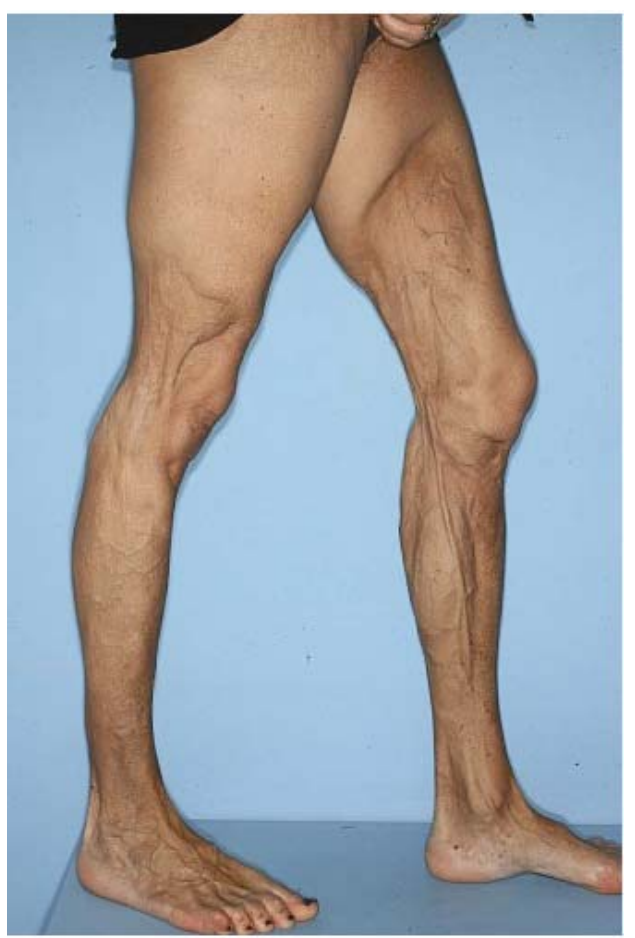

Figure 3. Diffuse and symmetric lipoatrophy from the root of thighs to the ankles of lower limbs

\section{Discussion}

Lipoatrophic panniculitis is a group of panniculitides that resolve leaving atrophic and depressed areas of the skin. These entities are divided into primary and secondary forms. Primary lipoatrophy is idiopathic, whereas secondary lipoatrophy may be because by various disorders such us $\alpha 1$-antitrypsin deficiency, pancreatic disease, infection, malignancy or connective tissue diseases as erythematosus panniculitis, subcutaneous morphea and panniculitis associated to dermatomyositis ${ }^{[1,2]}$. In our case, we did not find any proof to suggest an associated infectious process, pancreatic, connective tissue disease or malignancy.

Primary lipoatrophic panniculitis are not well described in the literature. It affects specially children and young women. This entity is firstly described by Winkelmann and Padilha-Goncalves in $1980^{[3]}$ as the "connective tissue panniculitis". The disease usually begins as an enlarging nodules and plaques on the trunk and extremities which resolve leaving several areas of localized lipoatrophy. The lesions may coalesce leaving extensive lipoatrophy. Auto-antibodies or auto-immune diseases (diabetes mellitus, Hashimoto thyroiditis, Graves disease...) are sometimes associated ${ }^{[4,5]}$. Histologically, biopsy specimens vary from a lymphohistiocytic lobular panniculitis to a lipophagic granulomatous panniculitis with numerous lipophagic histiocytes and Touton-like giants cells ${ }^{[4,5]}$. Treatment options include prednisone, antimalarial agents, saturated solution of potassium iodide, and dapsone with varying degrees of efficacy ${ }^{[4]}$. Our patient responded initially well to a combination of prednisone and hydroxychloroquine.

Lipoatrophic panniculitis is a diagnosis of exclusion that requires evaluating the patient for infection, autoimmune diseases and other causes of panniculitis. The most interesting features in our patient were the clinical characteristics. She developed a diffuse and symmetrical lipoatrophy after an extensive inflammatory process on the lower limbs that unfortunately progressed after treatment interruption. So, in case of clinical suspicion of lipoatrophic panniculitis, treatment must not be delayed. 
Dermatologist and dermatopathologist should be able to recognize this uncommon entity to prevent lipoatrophic sequalaes. Further studies are needed to determine the nosological context and treatment options of this progressive disease.

\section{References}

[1] Camacho D, Pielasinski U, Revelles JM, Gonzalez M, Haro R, Martin L et al. Diffuse lower limb lipoatrophy. J Cutan Pathol. 2011; 38: 270. http://dx.doi.org/10.1111/j.1600-0560.2010.01639.x

[2] Martinez A, Malone M, Hoeger P, Palmer R, Harper JI. Lipoatrophic panniculitis and chromosome 10 abnormality. Br J Dermatol. 2000; 142: 1034. http://dx.doi.org/10.1046/j.1365-2133.2000.03493.x

[3] Winkelmann RK, Padilha-Goncalves A. Connective tissue panniculitis. Arch Dermatol. 1980; 116: 291. http://dx.doi.org/10.1001/archderm.1980.01640270051012

[4] Shen LY, Edmonson MB, Williams GP, Gottam CC, Hinshaw MA, Teng JM. Lipoatrophic panniculitis: case report and review of the literature. Arch Dermatol. 2010; 146: 877-81. http://dx.doi.org/10.1001/archdermatol.2010.180

[5] Moulonguet I, Braun-Arduz P, Plantier F, Lerolle N, Petit A. Panniculite lipoatrophiante de l'adulte: traitement par hydroxychloroquine Ann dermatol venereal. 2011; 138: 681-5 